\title{
News, Announcements, and IASP
}

\section{Announcements}

The 2022 Virtual Conference held by Kevin's Song, called Equity \& Equality in Suicide Prevention, is taking place January 27-29, 2022. For more information go to http://kevinssong.org/conference/

The 55th Annual Conference of the American Association of Suicidology (AAS) is taking place April 27-30, 2022, in Chicago, IL, USA. For more information go to https://suicidology.org/conference/

The 10th International Association for Suicide Prevention (IASP) Asia Pacific Conference is taking place May 3-5, 2022, on the Gold Coast, QLD, Australia. For more information go to https://www.iasp.info/apac-home/
The 19th European Symposium of Suicide and Suicidal Behaviour is taking place August 24-27, 2022, in Copenhagen, Denmark. For more information go to http:// esssb19.org/

The National Suicide Prevention Conference held by Suicide Prevention Australia is taking place May 1-4, 2023, in Canberra, Australia. For more information go to https://www.suicidepreventionaust.org/our-events\#na tional-conference 


\section{International Association for Suicide Prevention}

\section{Would you like to join IASP?}

IASP is a nonprofit organization for those interested and working in suicide prevention. The membership consists of individuals (clinicians, scientists, crisis workers, volunteers and persons who have lost a family member by suicide) and community, national and international organizations. At this moment the membership extends over 50 countries and is affiliated with the World Health Organization as the key organization concerned with suicide prevention.

\section{The main objectives of IASP are:}

- to provide a common platform for all representatives of the many professions who are engaged in the field of suicide prevention and crisis intervention;

- to allow interchange of acquired experience in this area in various countries, especially through the exchange of literature;

- to promote the establishment of national organizations for suicide prevention;

- to facilitate the wider dissemination of the fundamentals of effective suicide prevention to professional groups and to the general public;

- to arrange for specialized training of selected persons in the area of suicide prevention in selected training centers;
- to carry out programs of research, especially those which can be pursued through international joint cooperation.

The activities of the association focus on the importance of sharing information, research and knowledge in order to address the issues of suicide and its prevention. The biennial congress, the bimonthly journal Crisis, biannual newsletter and the website serves as a medium for international exchange and a directory of members involved in suicidology is made available.

Candidates for IASP membership may apply directly to the Central Administration Office membership@iasp.info or join IASP online at http://www.iasp.info

Membership consists of:

- Regular membership for individuals regularly engaged in suicide studies, clinical management of suicidal patients, and/or suicide prevention activities, and who are interested in furthering the work of the Association.

- Organizational membership is for national or international voluntary or other nonprofit organizations (incorporated or unincorporated) working in the field of suicide studies, clinical management of suicidal patients and/or suicide prevention activities under the terms of their governing document.

- Associate membership is for individuals and organizations who are interested in working toward the goals of the Association, but who are not qualified for regular membership.

Please contact the IASP Central Administration Office at membership@iasp.info.; on the web at http://www.iasp.info

\section{IASP membership fees (include a subscription to Crisis)}

\begin{tabular}{|c|c|c|c|}
\hline Zone & Crisis & Individual Membership & Organizational Membership \\
\hline \multirow[t]{2}{*}{1} & Hard copy & US $\$ 190.00$ (early bird US $\$ 180.50,2$ yrs US $\$ 361.00,3$ yrs US $\$ 515.00$ ) & US $\$ 235.00$ (early bird US $\$ 225.00,2$ yrs US $\$ 446.50,3$ yrs US $\$ 635.00$ ) \\
\hline & Online only & US $\$ 162.00$ (early bird US $\$ 153.00,2$ yrs US $\$ 308.00,3$ yrs US $\$ 438.00$ ) & US $\$ 200.00$ (early bird US $\$ 190.00,2$ yrs US $\$ 380.00,3$ yrs US $\$ 543.00$ ) \\
\hline \multirow[t]{2}{*}{2} & Hard copy & US $\$ 160.00$ (early bird US $\$ 152.00,2$ yrs US $\$ 304.00,3$ yrs US $\$ 430.00$ ) & US $\$ 180.00$ (early bird US $\$ 170.00,2$ yrs US $\$ 342.00,3$ yrs US $\$ 485.00$ ) \\
\hline & Online only & US $\$ 136.00$ (early bird US $\$ 129.00,2$ yrs US $\$ 258.50,3$ yrs US $\$ 368.00$ ) & US $\$ 153.00$ (early bird US $\$ 145.00,2$ yrs US $\$ 290.50,3$ yrs US $\$ 414.00$ ) \\
\hline \multirow[t]{2}{*}{3} & Hard copy & US $\$ 135.00$ (early bird US $\$ 128.00,2$ yrs US $\$ 256.50,3$ yrs US $\$ 365.00$ ) & US $\$ 160.00$ (early bird US $\$ 150.00,2$ yrs US $\$ 304.00,3$ yrs US $\$ 430.00$ ) \\
\hline & Online only & US $\$ 115.00$ (early bird US $\$ 109.00,2$ yrs US $\$ 218.50,3$ yrs US $\$ 310.00$ ) & US $\$ 136.00$ (early bird US $\$ 129.00,2$ yrs US $\$ 258.50,3$ yrs US $\$ 368.00$ ) \\
\hline \multirow[t]{2}{*}{4} & Hard copy & US $\$ 115.00$ (early bird US $\$ 109.00,2$ yrs US $\$ 218.50,3$ yrs US $\$ 310.00$ ) & US $\$ 125.00$ (early bird US $\$ 120.00,2$ yrs US $\$ 237.50,3$ yrs US $\$ 340.00$ ) \\
\hline & Online only & US $\$ 98.00$ (early bird US $\$ 93.00,2$ yrs US $\$ 186.00,3$ yrs US $\$ 239.00$ ) & US $\$ 106.00$ (early bird US $\$ 101.00,2$ yrs US $\$ 201.50,3$ yrs US $\$ 288.00$ ) \\
\hline $\begin{array}{l}\text { All zones: } \\
\text { students, } \\
\text { volunteers, } \\
\text { \& retirees }\end{array}$ & Online only & US $\$ 115.00$ (early bird US $\$ 109.00,2$ yrs US $\$ 218.50,3$ yrs US $\$ 310.00$ ) & $\begin{array}{l}\text { For Advanced Organization Membership fees go to } \\
\text { http://www.iasp.info/application.php }\end{array}$ \\
\hline
\end{tabular}

\section{IASP Executive Committee 2021-2024}

President: $\quad$ Prof. Rory O'Connor (UK) 2021-2024

Vice President 1: Prof. Thomas Niederkrotenthaler (Austria) 2021-2024

Vice President 2: Prof. Jo Robinson (Australia) 2021-2022

Vice President 3: Prof. Lai Fong Chan (Malaysia) 2021-2022
General Secretary: Prof. Annette Erlangsen (Denmark) 2021-2024

Treasurer: $\quad$ Prof. Maurizio Pompili (Italy) 2021-2022

\section{IASP Council of National Representatives 2021-2024}

Co-Chairs: Prof. Charity Akotia (Ghana) 2021-2024 and Dr. Silvia Pelaez (Uruguay) 2021-2022

For a current listing of the IASP Council of National Representatives please refer to https://www.iasp.info/council_of_national_represent atives.php 